CLINICAL RESEARCH ARTICLE

\title{
Continuous glucose monitoring profile during therapeutic hypothermia in encephalopathic infants with unfavorable outcome
}

Paolo Montaldo ${ }^{1,2}$, Elisabetta Caredda ${ }^{1}$, Umberto Pugliese ${ }^{1}$, Angela Zanfardino ${ }^{3}$, Chiara Delehaye $^{1}$, Emanuela Inserra ${ }^{1}$, Laura Capozzi ${ }^{4}$, Giovanni Chello ${ }^{4}$, Carlo Capristo ${ }^{1}$, Emanuele Miraglia Del Giudice ${ }^{1}$ and Dario lafusco ${ }^{3}$

BACKGROUND: The relation between glucose homeostasis and outcome in hypoxic-ischemic encephalopathy (HIE) is unclear. To investigate whether glucose abnormalities assessed by using continuous interstitial glucose monitoring (CGM) correlate with later neurological outcomes in HIE.

METHODS: Prospective cohort study recruiting full-term neonates who received therapeutic hypothermia for HIE. CGM devices were placed soon after birth and recorded glucose profile for 3 days. The association between hypoglycemia ( $\leq 50 \mathrm{mg} / \mathrm{dL}$ ), hyperglycemia ( $>144 \mathrm{mg} / \mathrm{dL}$ ) and primary outcome defined as death or moderate or severe disability was examined with generalized estimating equations adjusted for Apgar scores, umbilical artery $\mathrm{pH}$ and base deficit. Neurodevelopmental outcome was assessed between 18 and 24 months.

RESULTS: Fifty-four neonates had outcome data available for the analysis; 19 of them (35\%) had adverse outcome. Longer duration of hypoglycemia (OR 7.1, 95\% Cl 1.8-20.3, $P<0.001$ ) and hyperglycemia (OR 5.4, 95\% Cl 1.6-15.7, $P<0.001)$, a greater area under the hypoglycemic (OR $2.6,95 \% \mathrm{Cl} 1.4-4.6, P=0.04)$ and hyperglycemic (OR 6.4, 95\% Cl 1.9-16.3, $P<0.001)$ curve were significantly associated with adverse outcomes.

CONCLUSION: Both hyper and hypoglycemia may be associated with adverse outcome in neonates with HIE. Future studies are needed to assess their prognostic association with neurological outcome.

Pediatric Research (2020) 88:218-224; https://doi.org/10.1038/s41390-020-0827-4

\section{IMPACT:}

- Glucose abnormalities during therapeutic hypothermia are associated with later neurological outcomes.

- Increased glucose variability correlates to the neurological outcome between 18 and 24 months.

- This study provides the first data on the continuous glucose profile in a group of HIE infants followed up to 2 years of age.

- Glucose homeostasis represents a key point in the management of HIE patients.

- Further research is needed to find the appropriate glycemic target in this population.

\section{INTRODUCTION}

Hypoxic ischemic encephalopathy (HIE) occurs in 2-4 per 1000 live births and is considered the most common cause of death and disability in term infants. ${ }^{1}$ Therapeutic hypothermia reduces the apparent brain injury on MR imaging, improves the survival rate and increases the likelihood of having a normal neurological outcome. Despite that, still up to $40 \%$ of infants with HIE go on to develop long-term disability. ${ }^{2}$ A variable response to therapeutic hypothermia probably reflects the complex pathophysiology of HIE (i.e. the uncertain severity, timing and patterns of the hypoxic insult). Increasing evidence shows that neonates with HIE are at a higher risk of both hypoglycemia and hyperglycemia and these glucose abnormalities are associated with brain injury on conventional MRI and adverse outcomes. ${ }^{3-5}$ However, there is still conflicting evidence regarding which glycemic threshold should be targeted and what is the best management of glucose instability in HIE.

Although serum glucose measurement is considered the gold standard in infants, glucose monitoring relies on intermittent heel pricks and frequently repeated sampling. Low glucose concentrations might not be detected by intermittent blood glucose monitoring and conversely blood glucose concentration may be assessed when the infant is only temporarily hypoglycemic, leading to unnecessary treatment. Continuous glucose monitoring (CGM) has the advantage of tracking glucose levels continuously and thus, it provides a reliable glycemic profile of patients. This device is currently used in the management of children with type 1 diabetes mellitus, leading to improved metabolic control in

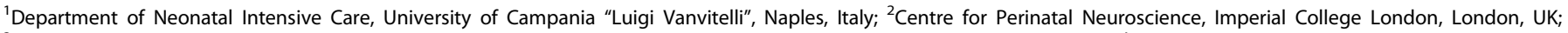

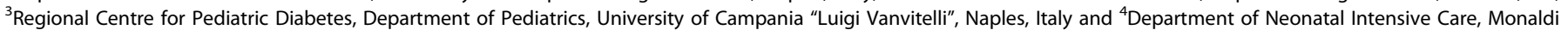
Hospital, Naples, Italy

Correspondence: Paolo Montaldo (p.montaldo@imperial.ac.uk) 
these patients. ${ }^{6}$ Nevertheless, its use in neonatal intensive care is limited due to paucity of data in newborns with only one study assessing the use of CGM in infants with HIE. ${ }^{7}$ The authors assessed the association between glucose control and amplitudeintegrated electroencephalography abnormalities. However, neurodevelopmental outcome was not evaluated.

A better understanding of the impact of glucose abnormalities on neurological outcomes may help to achieve a better intensive care management of neonates with HIE and in this way, enhance the efficacy of therapeutic hypothermia. Therefore, the objective of this study was to investigate for any association between glucose abnormalities assessed by using CGM and neurodevelopmental outcome between 18 and 24 months in infants with moderate or severe HIE treated with therapeutic hypothermia.

\section{METHODS}

This was a prospective longitudinal study conducted in the neonatal intensive care unit of the Monaldi Hospital, Naples, Italy. The study includes the patients recruited from January 2015 to July 2017 with moderate or severe neonatal encephalopathy, who fulfilled the TOBY trial entry criteria for therapeutic hypothermia. ${ }^{8}$ Exclusion criteria were suspected or confirmed congenital malformations or infections, inborn errors of metabolism or if CGM device could not be inserted within $6 \mathrm{~h}$ after birth.

The study protocol was approved by the ethics committee of the University of Campania "Luigi Vanvitelli" and informed written consent was obtained from parents or guardians of all participating neonates.

All the newborns were cooled within $6 \mathrm{~h}$ after birth to a core body temperature of $33.5^{\circ} \mathrm{C}$ for $72 \mathrm{~h}$ using a cooling blanket (Blanketrol II Hyper-Hypothermia System, Cincinnati Sub-Zero Products, Inc., Cincinnati, OH) followed by gradual rewarming (0.5 ${ }^{\circ} \mathrm{C}$ per hour) until body temperature reached $36.5^{\circ} \mathrm{C}$. Temperature was assessed by a rectal probe. Inotropic support was started by the attending clinician when mean arterial blood pressure was $<45 \mathrm{mmHg}$. Local protocol for cooling included the use of intravenous fentanyl during the whole duration of the therapeutic hypothermia treatment. After admission, infants were commenced on $40 \mathrm{ml} / \mathrm{kg} / 24 \mathrm{~h}$ Dextrose $15 \%$ infusion with basal glucose infusion rates of $4 \mathrm{mg} / \mathrm{kg} / \mathrm{min}$, which were then adjusted based on urine output, creatinine levels, electrolytes and glucose levels. Enteral nutrition support was deferred until rewarming phase.

Dexcom ${ }^{\circledast}$ G4 Platinum CGM sensor and transmitter (Dexcom, San Diego, CA, USA) were placed soon after study enrollment. The sensors were inserted in the lateral thigh and continuous measurements were recorded for $72 \mathrm{~h}$. The CGM device was calibrated using blood glucose values measured by point-of-care blood gas analyzer (Radiometer, ABL90 Flex, Copenhagen). CGM calibrations were performed at least twice a day as per the manufacturer's instructions. The CGM data were downloaded by using Dexcom Studio software on a dedicated computer.

Hypoglycemia was defined as blood or interstitial glucose $\leq 50 \mathrm{mg} / \mathrm{dL}(2.8 \mathrm{mmol} / \mathrm{L})$ and hyperglycemia as glucose $>144 \mathrm{mg} /$ $\mathrm{dL}(8.0 \mathrm{mmol} / \mathrm{L})$. Additional analysis was also performed using $60 \mathrm{mg} / \mathrm{dL}$ ( $3.3 \mathrm{mmol} / \mathrm{L})$ as cut-off of hypoglycemia after $48 \mathrm{~h}$ of age as recommended by the Pediatric Endocrine Society. ${ }^{9}$ When two or more consecutive data points $(\geq 10 \mathrm{~min}$ ) were outside the normal glucose range, these episodes were considered as interstitial glucose derangements. In case the episodes of glucose derangement were separated by short periods of normoglycemia lasting $<10 \mathrm{~min}$, they were considered as being continuous as previously described.

The clinical team was blinded to the CGM readings. Hypoglycemia and hyperglycemia were treated according to the local clinical guidelines of the newborn intensive care unit. Blood glucose values $<50 \mathrm{mg} / \mathrm{dL}(2.8 \mathrm{mmol} / \mathrm{L})$ were treated with intravenous dextrose boluses or increase of the glucose infusion rate. In case of hyperglycemia, the decision to start insulin infusion was made by the attending clinician as clinically indicated.

Neurodevelopment was assessed between 18 and 24 months by using the Bayley Scales of Infant and Toddler Development (Third Edition, Bayley-III) by trained personnel not involved in neonatal care. Adverse outcome was defined as a composite of death or moderate or severe disability according to the NICHD Neonatal Research Network criteria. ${ }^{10}$ Moderate disability was defined by a cognitive score from 70 to 84 and any of the following: a Gross Motor Function Classification System level of 2, an active seizure disorder (antiepileptic drugs in use), or a hearing deficit with the ability to follow commands after amplification. Severe disability was defined by any of the following: cognitive score $<70$, Gross Motor Function Classification System level $3-5$, blindness, or hearing impairment with inability to follow commands in spite of amplification. ${ }^{10}$ Where Bayley Scales of Infant Development III examination was not available, we used the components of severe disability as defined by the British Association of Perinatal Medicine (BAPM)/Royal College of Paediatrics and Child Health (RCPCH) working group classification. ${ }^{11}$

Statistical analyses

Demographic and clinical features are summarized by using descriptive statistics.

For each patient we calculated the mean, minimum, and maximum glucose levels based on CGM measurements obtained over the whole period of CGM recording $(72 \mathrm{~h})$. The area under the curve was calculated as the area of interstitial glucose concentration above or below the normal glucose range to assess the duration and severity of any interstitial glucose derangement episode. Standard deviation (SD), coefficient of variation (SD/ mean), and mean glucose rate of change per hour $(\mathrm{mg} / \mathrm{dL} / \mathrm{h})$ were used to quantify the degree of glucose variability. In order to identify whether a specific time interval after admission was most informative for predicting an adverse outcome, we also divided the period of recording (overall $72 \mathrm{~h}$ ) into 12 time intervals, each of $6 \mathrm{~h}$ starting from the time of admission of the neonate (Admission-6h, 6-12 h, 12-18 h, 18-24 h, 24-30 h, 30-36 h, 36-42 h, 42-48 h, 48-54 h, 54-60 h, 60-66 h, 66-72 h).

We used a generalized estimating equation with a binary dependent variable (adverse outcome yes/no), and glucose measures (mean, maximum or minimum glucose values, duration of glucose disturbance, and area under the curve) and measures of glucose variability (SD, coefficient of variation, and mean glucose rate of change per hour) as predictor variables. The glucose rate of change at a certain time was computed as described by Kovatchev et al. $^{12}$ The analysis was adjusted for encephalopathy stage at the time of admission, Apgar scores at 1, 5, 10 min, arterial cord $\mathrm{pH}$ and base excess. We used unstructured covariance matrix and binomial distribution with logit link function, resulting in odds ratios $(95 \%$ confidence intervals) as risk estimates. We also used the same model to estimate the association of glucose measures and measures of glucose variability on studied endpoints at each of the 12 different time intervals. Additional analysis was also performed in the time interval $48-72 \mathrm{~h}$ of age by using $60 \mathrm{mg} / \mathrm{dL}$ $(3.3 \mathrm{mmol} / \mathrm{L})$ as cut-off of hypoglycemia.

We estimated a sample size of 54 patients based on the following assumptions: (i) expected difference between two study groups of at least $11 \mathrm{mg} / \mathrm{dL}^{13}$, (ii) SD of $20 \mathrm{mg} / \mathrm{dL}$ for glucose values measured by using CGM in neonates ${ }^{14}$, (iii) a probability of 0.05 for a type I error, (iv) a probability of 0.2 for a type II error (i.e., a power of $80 \%$ ), and (v) adverse outcome incidence of $35 \%$. We increased the sample size to 60 in order to take into account a drop-out rate of $10 \%$. Every year there is an average of 30 infants with moderate or severe HIE who undergo therapeutic hypothermia at the Monaldi Hospital. Therefore, we estimated the study duration to be of 2 years and 6 months to achieve the targeted sample size. 


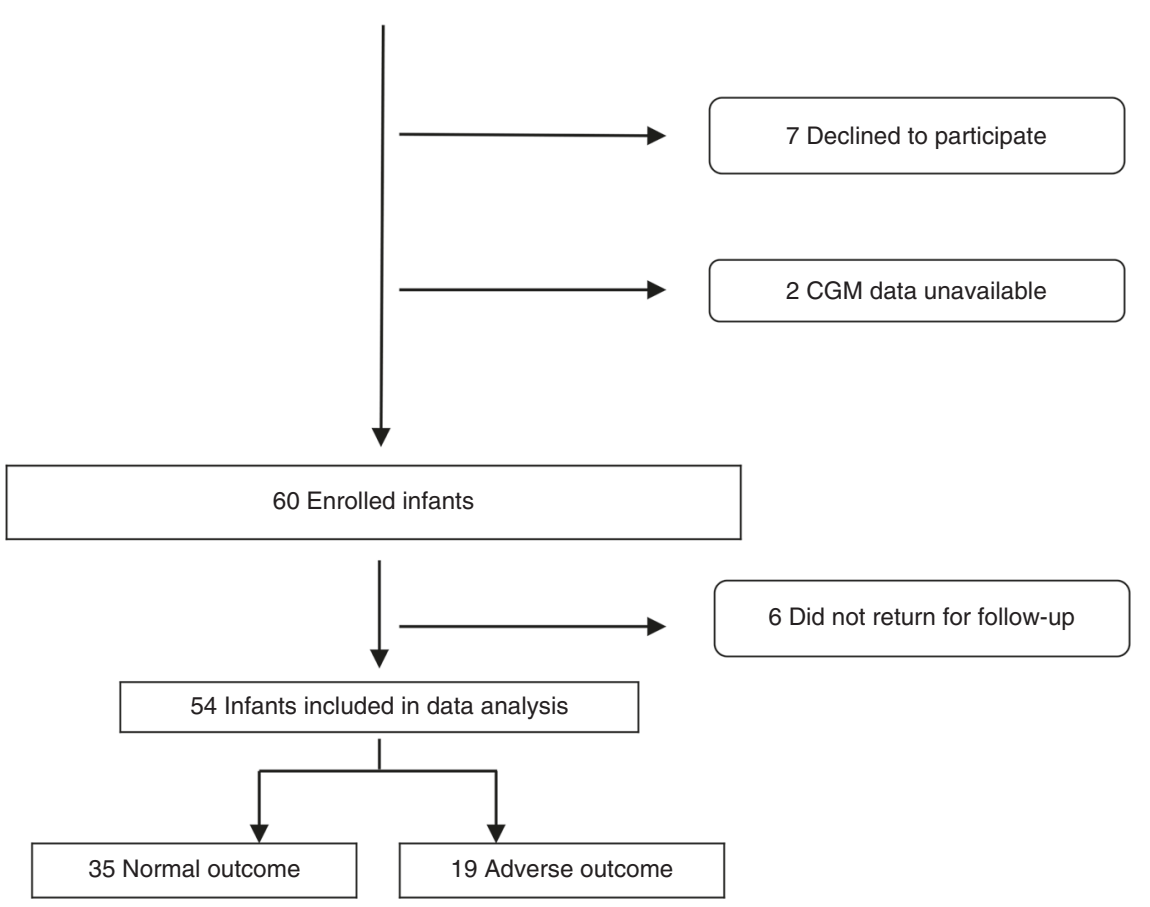

Fig. 1 Study profile. Patient recruitment and retention flow chart.

Two-tailed tests with $P$ value $<0.05$ were considered significant. We used SPSS Statistics 24 (IBM Corp., Armonk, NY) for statistical analyses.

\section{RESULTS}

During the study period, 69 families were approached to participate in the study, of whom seven refused to consent. In two cases CGM was not available for the analysis and six families did not return for follow-up. Thus, a total of 54 infants (78\%) were included in the study (Fig. 1). The average total fluid intake, urine output and glucose infusion rate during the $72 \mathrm{~h}$ of data collection were $58.9 \pm 9.3 \mathrm{~mL} / \mathrm{kg} / 24 \mathrm{~h}, 2.1 \pm 1.5 \mathrm{~mL} / \mathrm{kg} / \mathrm{h}$ and $5.4 \pm 0.9 \mathrm{mg} / \mathrm{kg} /$ min, respectively. Table 1 shows the characteristics of the infants.

Nineteen patients (35\%) had adverse outcome, of whom two died. In two out of the 19 children with adverse outcome, the Bayley assessment was not possible. Therefore, the components of disability, as defined by the BAPM/RCPCH, were considered for the outcome definition. In both the children, there was severe disability (Gross Motor Function Classification System >4) and in one of them there was also epilepsy, which prevented them from being assessed.

Overall, a mean of $59.1 \pm 11 \mathrm{~h}$ of CGM data were available per neonate. Thirty-seven (68\%) patients showed abnormal glucose values during the first $72 \mathrm{~h}$ after birth during CGM recording. Thirteen neonates had hypoglycemia (24\%), 16 neonates had hyperglycemia $(29 \%)$ and 8 neonates had both hypo- and hyperglycemia (15\%). One patient had an episode of hypoglycemia before insertion of the CGM. No data on glucose measurements outside the CGM recordings were included in the analysis.

Hypoglycemic episodes had a median duration of $82.1 \mathrm{~min}$ (IQR 40.1-133.2) lasting up to $205 \mathrm{~min}$. Hyperglycemic episodes had a median duration of $261.7 \mathrm{~min}$ (IQR 61.2-781) lasting up to $56.3 \mathrm{~h}$. During hypoglycemic episodes, the median interstitial glucose was $41 \mathrm{mg} / \mathrm{dL}$ ( $2.2 \mathrm{mmol} / \mathrm{L}$ ) (IQR 41-50, minimum $39 \mathrm{mg} / \mathrm{dL}$ ), and during hyperglycemic episodes, the median glucose was $248 \mathrm{mg} /$ $\mathrm{dL}$ (13.7 mmol/L) (IQR 192-368, maximum $398 \mathrm{mg} / \mathrm{dL})$. CGM
Table 1. Demographic, clinical characteristics of the patients. Data as median (range) or $n(\%)$.

\begin{tabular}{|c|c|c|c|}
\hline & $\begin{array}{l}\text { Good outcome } \\
(n=35)\end{array}$ & $\begin{array}{l}\text { Adverse outcome } \\
(n=19)\end{array}$ & $P$ value \\
\hline Female sex & $17(48)$ & $8(42)$ & 0.77 \\
\hline Gestational age (weeks) & $38.8(37-40)$ & $39.4(38-40)$ & 0.72 \\
\hline Birth weight (g) & $3215(3041-3513)$ & $3102(2900-3647)$ & 0.89 \\
\hline $\begin{array}{l}\text { HIE grade (Moderate/ } \\
\text { Severe) }\end{array}$ & $33 / 2$ & $11 / 8$ & 0.002 \\
\hline Apgar $10 \mathrm{~min}$ & $6(4-7)$ & $5(4-7)$ & 0.90 \\
\hline Worst $\mathrm{pH}$ by $\leq 1 \mathrm{~h}$ & $7(6.9-7.07)$ & $6.95(6.86-6.98)$ & 0.06 \\
\hline Need for inotropic support & $21(60)$ & $16(84)$ & 0.12 \\
\hline $\begin{array}{l}\text { Total fentanyl within } 72 \mathrm{~h} \\
\text { (mcg per } \mathrm{kg})\end{array}$ & $108(104-116)$ & $104(99-120)$ & 0.21 \\
\hline Seizures & $18(51)$ & $15(79)$ & 0.07 \\
\hline Invasive ventilation & $31(88)$ & $19(100)$ & 0.25 \\
\hline Maternal diabetes & $1(3)$ & $3(16)$ & 0.11 \\
\hline Type II diabetes mellitus & 0 & 0 & - \\
\hline Gestational diabetes & $1(3)$ & $3(16)$ & 0.11 \\
\hline Maternal hypertension & $4(11)$ & $2(10)$ & 1 \\
\hline $\begin{array}{l}\text { Maternal preeclampsia/ } \\
\text { eclampsia }\end{array}$ & $2(6)$ & 0 & 0.53 \\
\hline
\end{tabular}

glucose variables are shown in Table 2. The number of calibrations needed in infants who had hyper- or hypoglycemia and those who did not have any episodes of glucose derangements were not statistically significantly different, 2 (IQR 2-2) versus 2 (IQR $2-2)$ per day, $P>0.05$. Overall, six infants received treatment for hypoglycemia with intravenous dextrose boluses. Three infants received an insulin infusion for treatment of hyperglycemia.

We found that a longer duration of hypoglycemia and hyperglycemia and a greater area under the hyperglycemic and hypoglycemic curve were significantly associated with adverse 
Table 2. CGM glucose variables in the two groups of patients. Data as mean $\pm \mathrm{SD}$.

\begin{tabular}{|c|c|c|c|}
\hline & $\begin{array}{l}\text { Good outcome } \\
(n=35)\end{array}$ & $\begin{array}{l}\text { Adverse outcome } \\
(n=19)\end{array}$ & $P$ value \\
\hline Mean glucose level (mg/dL) & $81 \pm 16$ & $90 \pm 25$ & 0.25 \\
\hline Minimum glucose level (mg/dL) & $47 \pm 7$ & $44 \pm 6$ & 0.11 \\
\hline Area under curve of low glucose values ${ }^{\mathrm{a}}, \mathrm{h} \times \mathrm{mg} / \mathrm{dL}$ & $3.8 \pm 7$ & $27.7 \pm 34$ & 0.001 \\
\hline Area over curve of high glucose values ${ }^{\mathrm{b}}, \mathrm{h} \times \mathrm{mg} / \mathrm{dL}$ & $11.2 \pm 9$ & $207.2 \pm 105$ & 0.001 \\
\hline
\end{tabular}

Table 3. Generalized estimating equation models for association between severity of glucose derangements with the neurological outcome.

\begin{tabular}{|c|c|c|c|c|}
\hline & $\begin{array}{l}\text { Unadjusted OR } \\
(95 \% \mathrm{Cl})\end{array}$ & $P$ value & $\begin{array}{l}\text { Adjusted OR } \\
(95 \% \mathrm{Cl})\end{array}$ & $P$ value \\
\hline \multicolumn{5}{|l|}{ Hypoglycemia } \\
\hline Minimum glucose, $\mathrm{mg} / \mathrm{dL}$ & $2.02(0.7$ to 6.5$)$ & 0.45 & $2.6(0.89$ to 7.5$)$ & 0.36 \\
\hline Mean glucose, mg/dL & $1.4(0.6$ to 5.7$)$ & 0.31 & $1.8(0.74$ to 7.1$)$ & 0.20 \\
\hline Area under curve, $\mathrm{h} \times \mathrm{mg} / \mathrm{dL}$ & 3.1 (2.2 to 5.4$)$ & 0.001 & 2.6 (1.4 to 4.6$)$ & 0.04 \\
\hline Time hyperglycemic, h & $5.9(2.1$ to 14.2$)$ & $<.001$ & $5.4(1.6$ to 15.7$)$ & $<0.001$ \\
\hline Maximum glucose, $\mathrm{mg} / \mathrm{dL}$ & $3.7(1.7$ to 14.2$)$ & 0.02 & $2.6(1.4$ to 10.4$)$ & 0.01 \\
\hline Mean glucose, mg/dL & 2.7 (1.6 to 13.3$)$ & 0.04 & $2.3(1.3$ to 11.1$)$ & 0.02 \\
\hline Area under curve, $\mathrm{h} \times \mathrm{mg} / \mathrm{dL}$ & $5.0(1.8$ to 18.2$)$ & $<0.001$ & $6.4(1.9$ to 16.3$)$ & $<0.001$ \\
\hline \multicolumn{5}{|l|}{ Glucose variability } \\
\hline
\end{tabular}

outcome between 18 and 24 months. There was also a significant relationship between greater SD in interstitial glucose concentration and adverse outcome and between coefficient of variation (SD/mean) and adverse outcome (Table 3). The dispersion of glucose levels of both groups is shown in Fig. 2.

When we examined the different time periods, after $12 \mathrm{~h}$ of recording, we did not find any significant association between glucose derangements and 2-year outcome $(P>0.05)$ (Fig. 3). There was no statistically significant association between hypoglycemia and adverse outcome when we used $60 \mathrm{mg} / \mathrm{dL}$ ( 3.3 $\mathrm{mmol} / \mathrm{L}$ ) as cut-off of hypoglycemia in the time interval $48-72 \mathrm{~h}$ of age (Supplementary Table S1).

\section{DISCUSSION}

Our study showed that both hyperglycemia and hypoglycemia during early postnatal period were associated with adverse outcome after adjustment for some clinical markers of HIE. Moreover, increased glucose variability was also correlated to the neurological outcome between 18 and 24 months. This study provides the first data on the continuous glucose profile in a group of HIE infants followed up to 2 years of age and shows a relationship between glucose abnormalities during the $72 \mathrm{~h}$ of recordings and adverse outcome.
Glucose derangements in the early postnatal period have been previously investigated for their association with neurological outcomes in HIE neonates. However, our current knowledge is mainly based on retrospective studies or secondary analyses of randomized controlled trials, often, with conflicting results. $4,15,16$

A retrospective analysis of $52 \mathrm{HIE}$ infants who did not undergo therapeutic hypothermia showed that early hypoglycemia $(0-6 \mathrm{~h}$ of life), but not hyperglycemia was associated with adverse outcome. ${ }^{3}$ The secondary analysis of the CoolCap trial, instead, reported that both hypoglycemia and hyperglycemia were independently associated with adverse outcomes at 18 months. ${ }^{4}$ The same authors studied also the glycemic profile as predictor of the response to therapeutic hypothermia and showed that infants with early hyperglycemia were more likely to benefit from therapeutic hypothermia. ${ }^{15}$ On the contrary, Chouthai et al. reported poor outcome in hyperglycemic newborns undergoing therapeutic hypothermia. ${ }^{16}$

Hypoxic-ischemia increases the vulnerability of the brain to blood glucose concentrations, which are better tolerated by healthy neonates, suggesting that hypoxic-ischemic brain injury might even be exacerbated by concomitant glucose derangements. ${ }^{17}$ Therefore, glucose reference ranges for healthy term newborn may not be appropriate in HIE neonates where the hypoxic ischemic insult disturbs the normal postnatal metabolic 


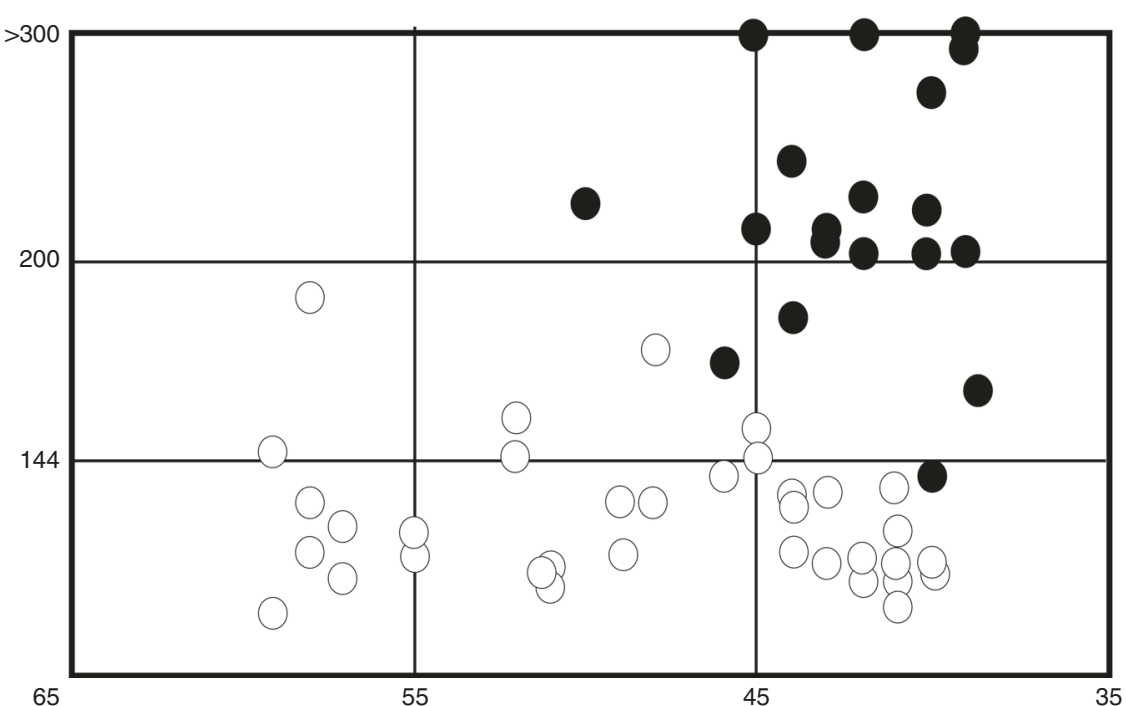

Fig. 2 Variability grid analysis. Each data point has blood glucose coordinates (minimum, maximum) for a subject during the considered time period of $72 \mathrm{~h}$. Infants with adverse outcome are shown as dots, infants with good outcome as open circles. The minimum blood glucose is plotted on the $x$-axis, which is inverse coded from 65 to $35 \mathrm{mg} / \mathrm{dL}$. The maximum blood glucose value is plotted on the $y$-axis.

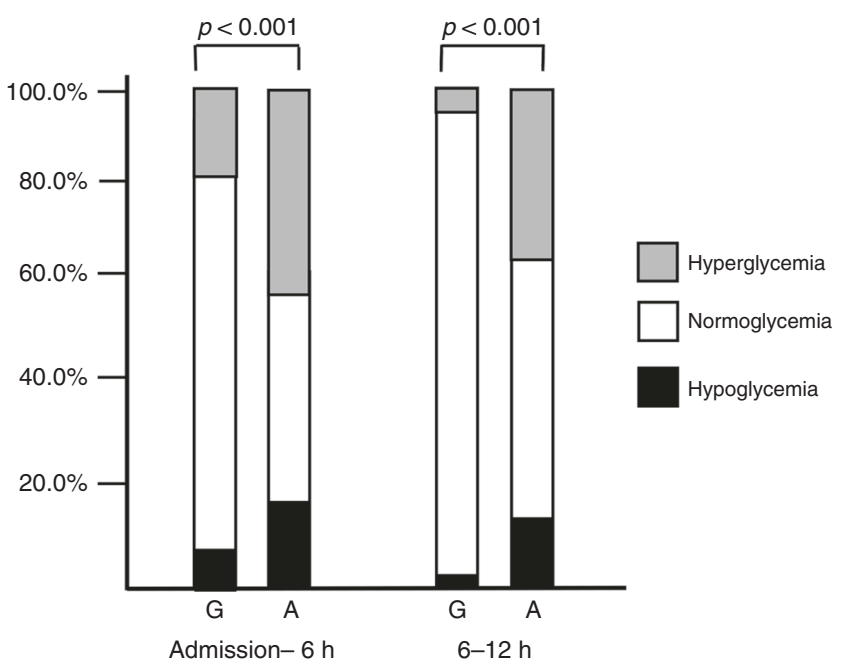

Fig. 3 Distribution of glucose values during the first $12 \mathrm{~h}$ of recording. Bar graphs compare the neurological outcome (G: good outcome, A: adverse outcome) with the percentage of time spent in normoglycemia, hyperglycemia and hypoglycemia.

transition and increases the odds of both hypoglycemia and hyperglycemia. ${ }^{4,18}$

Glucose abnormalities are common in asphyxiated neonates. Basu et al. showed that $57 \%$ of the infants recruited as part of the CoolCap trial had abnormal plasma glucose values within $12 \mathrm{~h}$ of randomization. ${ }^{4}$ Pinchefsky et al., more recently, reported that over $82 \%$ of the infants with HIE undergoing therapeutic hypothermia had abnormal glucose values in the first 3 days measured by using both interstitial and blood glucose values. ${ }^{7}$

Our findings confirm these data and show that almost $70 \%$ of neonates with HIE have glucose derangements, with most of them having hyperglycemia. More importantly, our study shows that the episodes of hyperglycemia lasted longer than those of hypoglycemia (median time 261.7 versus $82.1 \mathrm{~min}$ respectively). This might reflect a higher threshold for intervention in case of hyperglycemia and the tendency to tolerate higher glucose levels.

Increasing evidence suggests a possible link between hyperglycemia and neurological outcome. Preclinical studies showed that hyperglycemia after the hypoxic ischemic insult may cause a reduction in ATP brain storage and oxygen consumption, thus interfering with the recovery of brain cell membrane function and energy metabolism. ${ }^{19,20}$ However, clinical evidence is still controversial.

Recently, Pinchefsky et al. reported the first CGM data in HIE infants and showed that hyperglycemia, defined as glucose levels $>144 \mathrm{mg} / \mathrm{dL} \quad(8.0 \mathrm{mmol} / \mathrm{L})$, was associated with acute aEEG changes and seizure burden. Seizure burden is known to increase the risk of brain injury on magnetic resonance imaging, with a ninefold higher risk of adverse outcome if a neonate has a total seizure burden of more than $40 \mathrm{~min}^{21}$ Given the importance of these findings, we decided to use the same definition of hyperglycemia and in this way, we confirmed that hyperglycemia might be associated with later neurological outcomes.

In pediatric intensive care, hyperglycemia has been associated with adverse outcomes, including organ failure, length of stay and death. ${ }^{22,23}$ Although a tight glycemic control has been emerging as a possible strategy to improve outcomes, a systemic review and meta-analysis of the literature has failed to show any reduction of mortality through such control in critically ill children. ${ }^{23}$

The relation between glucose variability and mortality has been shown in children and adults. ${ }^{24,25}$ Moreover, a small retrospective study in HIE neonates highlighted that mean glucose variability during the first $24 \mathrm{~h}$ was significantly associated with the 2-year neurodevelopmental outcome. ${ }^{13}$ Our study supports these findings and suggests an association between hyperglycemia and glucose variability with adverse neurological outcome.

Hypoglycemia is known to worsen the effect of hypoxia on brain glucose reserves, by attenuating anaerobic glycolysis thus reducing high-energy phosphates. It also increases the vulnerability of watershed regions to ischemia, by impairing cerebrovascular autoregulation. ${ }^{26}$ Different studies have suggested that neonates with HIE may require higher concentrations of glucose to satisfy increased metabolic needs and have shown that a blood glucose concentration of less than $45 \mathrm{mg} / \mathrm{dL}$ ( $2.5 \mathrm{mmol} / \mathrm{L})$ may be a risk factor for a poor outcome. ${ }^{17}$ However, there are no data showing which glycemic concentration can provide optimal glucose delivery to the brain after HIE.

At the moment, there is considerable debate with regard to the definition of hypoglycemia and different thresholds were used in different guidelines and research studies. ${ }^{4,7}$ Evidence from neurophysiological and neurodevelopmental outcome studies 
has been mainly based on a threshold of $46 \mathrm{mg} / \mathrm{dL}(2.6 \mathrm{mmol} /$ L). ${ }^{27,28}$ However, more recently, the Pediatric Endocrine Society recommended that the goal in high-risk infants should be to maintain plasma glucose levels $>50 \mathrm{mg} / \mathrm{dL}(2.8 \mathrm{mmol} / \mathrm{L})$ in the first $48 \mathrm{~h}$ of age, which is also considered the threshold for neuroglycopenic symptoms. ${ }^{9}$ Accordingly, we decided to use $50 \mathrm{mg} / \mathrm{dL}$ ( $2.8 \mathrm{mmol} / \mathrm{L}$ ) as threshold for hypoglycemia in our study.

Our findings show that the time spent under hypoglycemic condition was significantly associated with the 2-year neurological outcome, but not the minimum glucose nor the mean glucose values. Although these data may suggest that the duration of the hypoglycemic episodes, more than the actual glucose values, is related to the neurological outcome, caution is needed before drawing further conclusions. In fact, CGM technology has some limits in the detection of glycemic values below $40 \mathrm{mg} / \mathrm{dL}$, despite different calibration strategies. ${ }^{29}$

After $48 \mathrm{~h}$ there is a maturation of glucose-stimulated insulin secretion, which leads to an increase of the mean glucose concentration by $72 \mathrm{~h}$ of age to values close to those in children. ${ }^{9}$ Therefore, a higher threshold of $60 \mathrm{mg} / \mathrm{dL}(3.3 \mathrm{mmol} / \mathrm{L})$ has been recently recommended for hypoglycemic definition after $48 \mathrm{~h}$ of age. ${ }^{9}$ Based on that, we analyzed the time interval $48-72 \mathrm{~h}$ of age also with a higher glycemic cut-off. However, no significant association has been found with the neurological outcome, which might reflect the progressive improvement of glucose homeostasis in infants with moderate or severe HIE as suggested by Basu et al. in the secondary analysis of CoolCap trial. ${ }^{4}$

In HIE neonates, hypoglycemia, hyperglycemia and glucose variability are potentially modifiable risk factors, and, therefore, there is growing interest in finding neuroprotective glucosemanagement strategies, since the optimal management of these conditions might significantly improve the outcome. Although different studies have shown the correlation between postnatal glucose profile and neurological outcomes, blood glucose measurements were performed through intermittent blood sampling. Intermittent blood glucose monitoring not only causes pain-related stress, but also may leave epochs of hypoglycemia and hyperglycemia undetected, thus exposing asphyxiated neonates to wide fluctuations in blood glucose.

Our study has strengths and limitations. Firstly, we could not investigate whether glucose derangements are the cause of brain injury or simply, expression of the extent of brain damage, even though our analysis was adjusted for some HIE severity markers. Secondly, although this is the first study comparing CGM glucose profile with neurological outcome in HIE, our small sample size can only provide data for future powered studies. Thirdly, we used the components of severity of the BAPM/RCPCH working group classification whereas Bayley-III was not available. Neurodevelopmental assessment should be performed in all the study participants. However, if a child is unable to complete the Bayley assessment because of severe disability, like in our cases, BAPM classification is used for outcome definition. ${ }^{30-32}$ Finally, CGM technology may show large errors when blood glucose concentration is falling. However, we performed multiple calibrations with accurate glucose measurements (blood gas analyzer) in order to improve its accuracy, as recently suggested. ${ }^{29}$ In future studies, point-to-point recalibration of the raw signal is recommended for retrospective analysis of the data in order to improve the accuracy of CGM in the low glucose range. ${ }^{29}$

Our data show that undetected hyperglycemia as well as hypoglycemia might be associated with adverse outcome with important implications for the clinical care. However, given its observational design, this study cannot infer causality between glucose derangements and brain injury. Our findings stress the importance of glucose homeostasis in HIE and highlight the need for further research to find the appropriate glycemic target in this population.

\section{AUTHOR CONTRIBUTIONS}

P.M., E.M.D.G., D.I. conceptualized and designed the study. P.M., E.C., and U.P. collected the data. A.Z., C.D. and E.I. analyzed the data. P.M., E.C., U.P. drafted the initial manuscript. D.I. supervised the study. L.C., G.C., C.C., E.M.D.G. and D.I. reviewed and revised the manuscript critically. All authors approved the final manuscript as submitted.

\section{ADDITIONAL INFORMATION}

The online version of this article (https://doi.org/10.1038/s41390-020-0827-4) contains supplementary material, which is available to authorized users.

Competing interests: The authors declare no competing interests.

Patient consent: All the parents of the neonates who were enrolled in the study provided their written informed consent.

Publisher's note Springer Nature remains neutral with regard to jurisdictional claims in published maps and institutional affiliations.

\section{REFERENCES}

1. Lawn, J., Shibuya, K. \& Stein, C. No cry at birth: global estimates of intrapartum stillbirths and intrapartum-related neonatal deaths. Bull. World Health Organ. 83, 409-417 (2005).

2. Jacobs, S. E. et al. Cooling for newborns with hypoxic ischaemic encephalopathy. Cochrane Database Syst. Rev. 1, Cd003311 (2013).

3. Nadeem, M., Murray, D. M., Boylan, G. B., Dempsey, E. M. \& Ryan, C. A. Early blood glucose profile and neurodevelopmental outcome at two years in neonatal hypoxic-ischaemic encephalopathy. BMC Pediatr. 11, 10 (2011).

4. Basu, S. K. et al. Hypoglycaemia and hyperglycaemia are associated with unfavourable outcome in infants with hypoxic ischaemic encephalopathy: a post hoc analysis of the CoolCap Study. Arch. Dis. Child Fetal Neonatal Ed. 101, F149-F155 (2016).

5. Basu, S. K. et al. Early glycemic profile is associated with brain injury patterns on magnetic resonance imaging in hypoxic ischemic encephalopathy. J. Pediatr. 203, 137-143 (2018).

6. Raviteja, K. V., Kumar, R., Dayal, D. \& Sachdeva, N. Clinical efficacy of Professional Continuous Glucose Monitoring in improving glycemic control among children with Type 1 diabetes mellitus: an open-label randomized control trial. Sci. Rep. $\mathbf{9}$ 6120 (2019).

7. Pinchefsky, E. F. et al. Hyperglycemia and glucose variability are associated with worse brain function and seizures in neonatal encephalopathy: a prospective cohort study. J. Pediatr. 209, 23-32 (2019).

8. Azzopardi, D. V. et al. Moderate hypothermia to treat perinatal asphyxial encephalopathy. N. Engl. J. Med. 361, 1349-1358 (2009).

9. Thornton, P. S. et al. Recommendations from the Pediatric Endocrine Society for Evaluation and Management of Persistent Hypoglycemia in Neonates, Infants, and Children. J. Pediatr. 167, 238-245 (2015).

10. Shankaran, S. et al. Effect of depth and duration of cooling on death or disability at age 18 months among neonates with hypoxic-ischemic encephalopathy: a randomized clinical trial. JAMA 318, 57-67 (2017).

11. BAPM/RCPCH Working Group. Classification of health status at 2 years as a perinatal outcome. http://www.bapm.org/publications/documents/guidelines/ OutcomeBAPMWG report v6 Jan08.pdf (2008).

12. Kovatchev, B. P., Clarke, W. L., Breton, M., Brayman, K. \& McCall, A. Quantifying temporal glucose variability in diabetes via continuous glucose monitoring: mathematical methods and clinical application. Diabetes Technol. Ther. 7, 849-862 (2005).

13. Al Shafouri, N., Narvey, M., Srinivasan, G., Vallance, J. \& Hansen, G. High glucose variability is associated with poor neurodevelopmental outcomes in neonatal hypoxic ischemic encephalopathy. J. Neonatal Perinat. Med. 8, 119-124 (2015).

14. Galderisi, A. et al. Continuous glucose monitoring in very preterm infants: a randomized controlled trial. Pediatrics 140, pii: e20171162 (2017).

15. Basu, S. K., Salemi, J. L., Gunn, A. J. \& Kaiser, J. R. Hyperglycaemia in infants with hypoxic-ischaemic encephalopathy is associated with improved outcomes after therapeutic hypothermia: a post hoc analysis of the CoolCap Study. Arch. Dis. Child Fetal Neonatal Ed. 102, F299-F306 (2017).

16. Chouthai, N. S. et al. Hyperglycemia is associated with poor outcome in newborn infants undergoing therapeutic hypothermia for hypoxic ischemic encephalopathy. J. Neonatal Perinat. Med. 8, 125-131 (2015).

17. Boardman, J. P. \& Hawdon, J. M. Hypoglycaemia and hypoxic-ischaemic encephalopathy. Dev. Med. Child Neurol. 57, 29-33 (2015).

18. Harding, J. E., Harris, D. L., Hegarty, J. E., Alsweiler, J. M. \& McKinlay, C. J. An emerging evidence base for the management of neonatal hypoglycaemia. Early Hum. Dev. 104, 51-56 (2017). 
19. Park, W. S., Chang, Y. S. \& Lee, M. Effects of hyperglycemia or hypoglycemia on brain cell membrane function and energy metabolism during the immediate reoxygenation-reperfusion period after acute transient global hypoxia-ischemia in the newborn piglet. Brain Res. 901, 102-108 (2001).

20. Lin, B., Ginsberg, M. D. \& Busto, R. Hyperglycemic exacerbation of neuronal damage following forebrain ischemia: microglial, astrocytic and endothelial alterations. Acta Neuropathol. 96, 610-620 (1998).

21. Kharoshankaya, L. et al. Seizure burden and neurodevelopmental outcome in neonates with hypoxic-ischemic encephalopathy. Dev. Med. Child Neurol. 58, 1242-1248 (2016).

22. Preissig, C. M. \& Rigby, M. R. Pediatric critical illness hyperglycemia: risk factors associated with development and severity of hyperglycemia in critically ill children. J. Pediatr. 155, 734-739 (2009).

23. Chen, L., Li, T., Fang, F., Zhang, Y. \& Faramand, A. Tight glycemic control in critically ill pediatric patients: a systematic review and meta-analysis. Crit. Care 22, 57 (2018).

24. Hirshberg, E., Larsen, G. \& Van Duker, H. Alterations in glucose homeostasis in the pediatric intensive care unit: hyperglycemia and glucose variability are associated with increased mortality and morbidity. Pediatr. Crit. Care Med. 9, 361-366 (2008).
25. Takahashi, H. et al. Glycemic variability determined with a continuous glucose monitoring system can predict prognosis after acute coronary syndrome. Cardiovasc. Diabetol. 17, 116 (2018)

26. Vannucci, R. C., Nardis, E. E. \& Vannucci, S. J. Cerebral metabolism during hypoglycemia dn asphyxia in newborn dogs. Biol. Neonate 38, 276-286 (1980).

27. Koh, T. H., Aynsley-Green, A., Tarbit, M. \& Eyre, J. A. Neural dysfunction during hypoglycaemia. Arch. Dis. Child. 63, 1353-1358 (1988).

28. Lucas, A., Morley, R. \& Cole, T. J. Adverse neurodevelopmental outcome of moderate neonatal hypoglycaemia. BMJ 297, 1304-1308 (1988).

29. Shah., R., McKinlay, C. J. D. \& Harding, J. E. Neonatal hypoglycemia: continuous glucose monitoring. Curr. Opin. Pediatr. 30, 204-208 (2018).

30. Lally, P. J. et al. Residual brain injury after early discontinuation of cooling therapy in mild neonatal encephalopathy. Arch. Dis. Child. Fetal Neonatal Ed. 103, F383-F387 (2018).

31. Lally, P. J. et al. Magnetic resonance spectroscopy assessment of brain injury after moderate hypothermia in neonatal encephalopathy: a prospective multicentre cohort study. Lancet Neurol. 18, 35-45 (2019).

32. Wood, N. S., Marlow, N., Costeloe, K., Gibson, A. T. \& Wilkinson, A. R. Neurologic and developmental disability after extremely preterm birth. EPICure Study Group. N. Engl. J. Med. 343, 378-384 (2000). 